\title{
Amplification by Pulses Interpolation with High Frequency Restriction for the conservation of the Structural Similarity of the Image
}

\author{
Leandro Morera Delfín \\ Higher Polytechnic Institute Jose Antonio Echeverria Electrical Engineering \\ Faculty,Telecommunications' Department. \\ leandro@electrica.cujae.edu.cu
}

\begin{abstract}
This article provides a comparison between methods of images amplification with good results in the preservation of the structural similarity of the output image. This parameter is evaluated through the method SSIM (structural similarity index).One of the methods used for amplification is the combination of SWT (stationary wavelet transform) for the decomposition of the image, SVD (singular value decomposition) for conservation of high frequency content and DWT ( discrete Wavelet Transform ) for obtain the high resolution image. This method has report of good results [5]. Other amplification technique is proposed, RHPI (pulses interpolation constrained by high frequency)[9].The procedure RHPI uses a theoretical model of digital image registration and high frequency filters for the construction of the interpolation kernel for the amplification process. Finally, both methods are compared through SSIM structural similarity coefficient and PSNR parameters. The proposed method RHPI is a solution better than the SWT-SVD-DWT for the improvement in the conservation of SSIM and PSNR ratios for high rates of amplification.
\end{abstract}

Keywords: Structural Similarity, interpolation pulses, Discrete Wavelet Transform, Singular Value Decomposition

\section{Introduction}

In the image processing, resolution and contrast are fundamental aspects [6]. The process most commonly used to increase the resolution of the images is the interpolation [7]. There is a close relationship between these parameters due to the interpolation process; this affects the frequency content of the image in the contours. The information of contours and fine details of the image must be transferred to the plane of high resolution in areas of the highest spatial frequency .The structural relationship of the pixels of the image should does not suffer significant changes due the amplification process. The contrast is related to the ability of the transference system applied to the image for detect a great number of changes in the values of the pixels in a defined range, this enhances the edges definition .Applying SVD, the singular value matrix [2] of an image contains luminance information and changing singular values directly affects the lighting of the picture. The stationary wavelet transform SWT decomposes the image bands of different types of information, these are matrices A, H, V, D Trend A, fluctuation in the horizontal $\mathrm{H}$, vertical and diagonal, V and D. The SWT [8] maintains the size of the image in each of these planes of information, however the DWT [8] Discrete Wavelet Transform incurs in a decimation process to obtain these matrices. That is why the method is used to obtain the high resolution image using the reverse process as input of the IDWT the result of SWT, the SVD is applied for maintain the contrast of the original image. The other method used in the comparison studies the high frequency content for the construction of an interpolation kernel for the amplification. This proposed procedure is the RHPI [9] (high frequency restriction for amplification by pulses interpolation) using a Canny filter [4] for the guide in the construction of the amplification kernel. This amplification kernel is extracted of an physical model of images scan. In this case the interpolation kernel dimensions are oriented by the high frequency content of the image of high resolution. 


\section{Amplification Methods Proposed}

The first method used is the combination of the transformed wavelet SWT, compensation of singular values SVD and inverse DWT for obtain the high resolution image. The transformed wavelet packet are applied using the Daubechies1.On each region's output is calculated the wavelet transform and singular value matrix S through SVD decomposition. For DWT the SVD decomposition is:

$$
\begin{aligned}
& A_{d w t}=U a_{d w t} \cdot S a_{d w t} \cdot V a_{d w t}{ }^{T} \\
& V_{d w t}=U v_{d w t} \cdot S v_{d w t} \cdot V v_{d w t}{ }^{T} \\
& H_{d w t}=U h_{d w t} \cdot S h_{d w t} \cdot V v_{d w t}{ }^{T} \\
& D_{d w t}=U d_{d w t} \cdot S d_{d w t} \cdot V d_{d w t}{ }^{T}
\end{aligned}
$$

And for SWT:

$$
\begin{aligned}
& A_{s w t}=U a_{s w t} \cdot S a_{s w t} \cdot V a_{s w t}{ }^{T} \\
& V_{s w t}=U v_{s w t} \cdot S v_{s w t} \cdot V v_{s w t}{ }^{T} \\
& H_{s w t}=U h_{s w t} \cdot S h_{s w t} \cdot V v_{s w t}{ }^{T} \\
& D_{s w t}=U d_{s w t} \cdot S d_{s w t} \cdot V d_{s w t}{ }^{T}
\end{aligned}
$$

The parameters $S a_{d w t}$ and $S a_{s w t}$ are output matrices of singular values, trends of the transformed SWT and DWT respectively. The parameters $U a_{d w t}$ and $U a_{s w t}$ are orthogonal matrices of the output, trends of the transformed

SWT and DWT respectively. The parameters $V a_{d w t}{ }^{T}$ and $V a_{s w t}{ }^{T}$ are orthogonal matrices are transposes of the trend output of the transformed DWT and SWT respectively. Similarly is defined the decomposition of the output matrices of the wavelet decomposition $\mathrm{H}, \mathrm{V}$ and $\mathrm{D}$. The correction coefficient is calculated from singular values in each matrix of the decomposition as:

$$
\Delta=\left(\frac{\max \left(S_{d w t}\right)}{\max \left(S_{s w t}\right)}\right)
$$

Is calculated a new matrix of singular values for each output matrix decomposition SWT. The Figure 1 shows the Structural diagram of this interpolation method. 


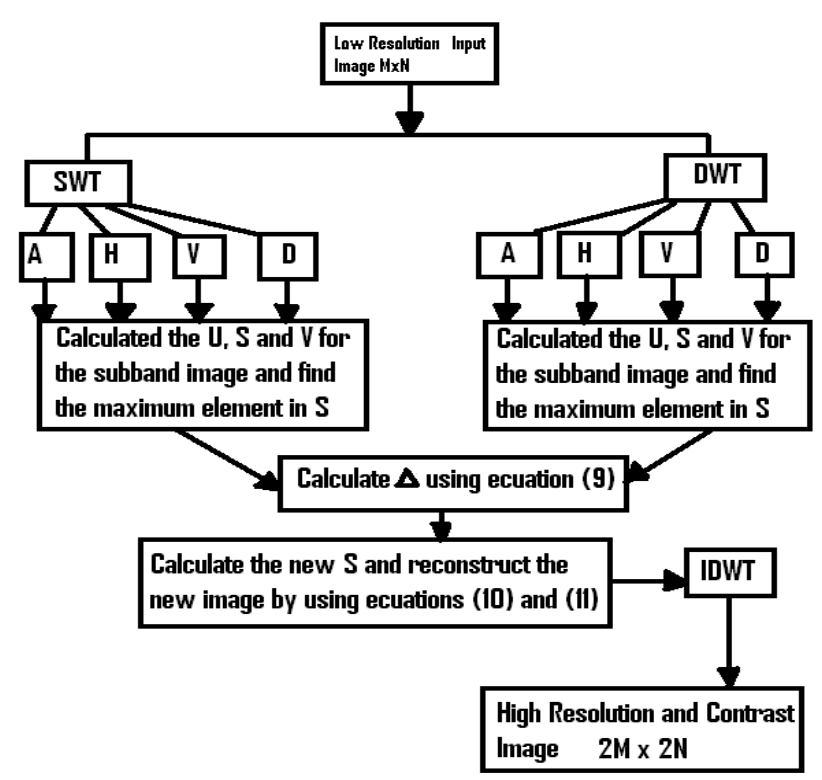

Figure 1. Structural Diagram of the Interpolation SWT-SVD-IDWT for the First Iteration

$S e_{s w t}=\Delta \cdot S_{s w t}$

This change each matrix A, H, V and D of the SWT decomposition with the new matrix of singular values corresponding to each plane.

$$
A_{s w t}=U_{s w t} \cdot S e_{s w t} \cdot V_{s w t}{ }^{T}
$$

The result is applied as input to the IDWT for build the high-resolution image. This procedure increase the resolution of the image size in a $2^{n}$ factor where $n$ is the iteration of the procedure.

The second method proposed, RHPI, is a restricted interpolation system made with spatial and window filters in order to increase the high frequency content at the high resolution image. The proposed method constructs an interpolation kernel using the high frequency content at high-resolution image domain as an explicit driving force for the variation of the amplification kernel parameters. With this process, the optimal low-high resolution pair is found using the amplification kernel given by the Fourier transform of the truncated sampling arrangement [10].The spectrum of high-resolution image is given by:

$$
F_{P}\left(w_{x}, w_{y}\right)=\left(\frac{1}{4 \pi^{2}}\right) F\left(w_{x}, w_{y}\right) *\left[D_{T}\left(w_{x}, w_{y}\right) \cdot P\left(w_{x}, w_{y}\right)\right]
$$

The Fourier transform of the truncated sampling arrangement [10] is:

$$
D_{T}\left(w_{x}, w_{y}\right)=\frac{\sin \left\{w_{x}\left(j+\frac{1}{2}\right) \cdot \Delta x\right\} \cdot \sin \left\{w_{y}\left(k+\frac{1}{2}\right) \cdot \Delta y\right\}}{\sin \left\{w_{x} \cdot \frac{\Delta x}{2}\right\} \cdot \sin \left\{w_{y} \cdot \frac{\Delta y}{2}\right\}}
$$

The space of band limited functions in the frequency range is traversed by the infinite set (but countable) sinc functions shifted by integers. Therefore any such band limited function $g(t)$ can be reconstructed from its samples with integer spacing. 


$$
g(t)=\sum_{n=-\infty}^{n=\infty} g(n) \cdot \sin c(t-n)
$$

Taken (12) and (14) is obtained:

$$
G_{P}\left(w_{x}, w_{y}\right)=\left(\frac{1}{4 \pi^{2}}\right) G\left(w_{x}, w_{y}\right) *\left[D_{T}\left(w_{x}, w_{y}\right) \cdot P\left(w_{x}, w_{y}\right)\right]
$$

Applying the convolution yields the high resolution spectrum Qc matrix described in Figure 2.The dimensions of the kernel of interpolation are restricted for the amplification by the high frequency content achieved at the output of the interpolation system. Is possible to use a kernel of convolution [3, 12].operating in the manner of the matrix Hc shown in the Figure 2.

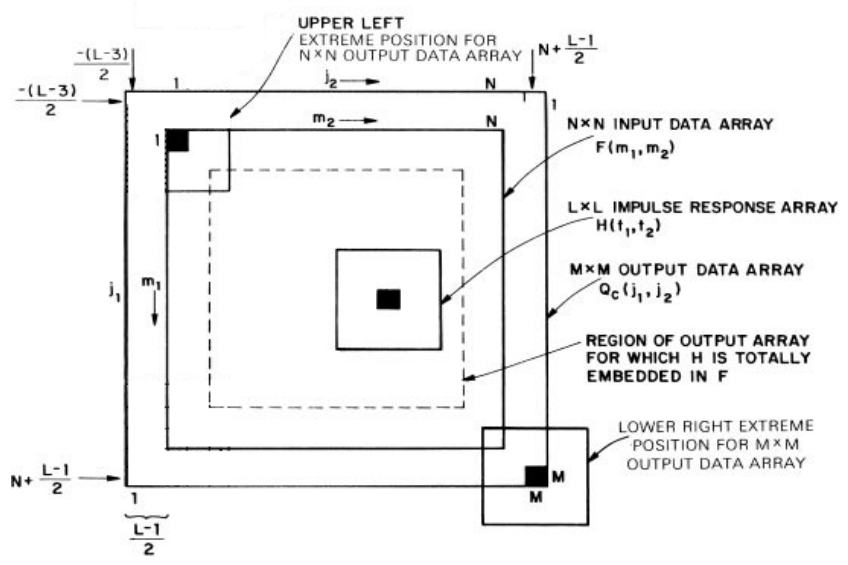

Figure 2. Relationships between Input Data, Output Data, and Impulse Response Arrays for Finite-Area Superposition; Centered Array Definition Taken from [11]

Applying the inverse Fourier transform to the array output data the high-resolution image is obtained.

$Q_{S}(x, y)=\sum_{\omega_{x}=-M_{x} / 2}^{\omega_{x}=M_{x} / 2} \sum_{\omega_{y}=-M_{y} / 2}^{\omega_{y}=M_{y} / 2} Q_{c}\left(\omega_{x}, \omega_{y}\right) \cdot \exp \left(j\left(\omega_{x} x+\omega_{y} y\right)\right)$

A Canny filter [4] is applied in order to estimate the amount of edges and restrict the interpolation kernel dimensions to this estimate. A Canny filter is applied.

$Q_{\text {SCanny }}(x, y)=\operatorname{Canny}\left\{Q_{S}(x, y)\right\}$

Then, the appropriate values of Lx and Ly of the impulse response array of the Figure 2 can be found through:

$Q_{\text {SCanny }}\left(\omega_{x}, \omega_{y}, L_{x}, L_{y}\right)=Q_{c}\left(j_{1}, j_{2}\right)$

$Q_{\text {Sop }}\left(L_{x}, L_{y}\right)=\sum_{x=-M_{x} / 2} \sum_{y=-M_{y} / 2} \sum_{\omega_{x}=-M_{x} / 2}^{M_{x} / 2} \sum_{\omega_{y}=-M_{y} / 2}^{y=M_{y} / 2} Q_{S f}\left(\omega_{x}, \omega_{y}, L_{x}, L_{y}\right) \cdot \exp \left(-j\left(\omega_{x} x+\omega_{y} y\right)\right)$

$\operatorname{MAX}\left(Q_{\text {Sop }}\left(L_{x}, L_{y}\right)\right)$ 


$$
\left(\frac{\partial^{2} Q_{S o p}\left(L_{x}, L_{y}\right)}{\partial L_{x} \partial L_{y}}\right)=0
$$

Lx and Ly are the dimensions of the amplification kernel. The solution of Equation 21 provides the optimum dimension for the interpolation kernel. Then the optimal low-high resolution pair for an amplification rate is found.

\section{Results Obtained}

In the proposed experiment an input image of $384 \times 512$ pixels is used. A comparative method is applied using the structural similarity SSIM and PSNR relationships. The method SWTDWT-SVD is applied for iterations $n=1$ and $n=2$.The RHPI method for optimal interpolation kernel is applied and the output image of major resolution is of $811 \times 1080$ pixels. The measurement method SSIM, structural similarity, measures the similarity between two images. This index is a comprehensive measure and benchmark to measure the quality of the image from a reference image without compression or distortion. The SSIM index improves the traditional measurement methods PSNR (peak power ratio of signal to noise level) and mean square error (MSE) which are inconsistent for ocular perception [13]. SSIM method considers the degradation of the image as changes in the structural information of the image. It is based on the idea that the pixels have strong structural interdependence when they are close. This dependence has important information about the structure of objects in the image.

SSIM index calculation for two image areas of the same size is calculated as follows:

$$
\begin{aligned}
& \operatorname{SSIM}(x, y)=\frac{\left(2 \cdot \mu_{x} \cdot \mu_{y}+c_{1}\right)\left(2 \cdot \sigma_{x y}+c_{2}\right)}{\left(\mu_{x}{ }^{2}+\mu_{y}{ }^{2}+c_{1}\right)\left(\sigma_{x}{ }^{2}+\sigma_{y}{ }^{2}+c_{2}\right)} \\
& M \operatorname{SSIM}=\frac{1}{M} \cdot \sum_{j=1}^{M} \operatorname{SSIM}\left(x_{j}, y_{j}\right)
\end{aligned}
$$

In these expressions ${ }^{\mu_{x}}$ and ${ }^{\mu_{y}}$ are averages of $\mathrm{x}$ and $\mathrm{y},{ }^{\sigma_{x}}{ }^{2}$ and ${ }^{\sigma_{y}{ }^{2}}$ are variances of $\mathrm{x}$ and $\mathrm{y}$, and ${ }^{\sigma_{x \cdot y}}$ is the covariance of $\mathrm{x}$ and $\mathrm{y}$. The terms $c_{1}=\left(K_{1} \cdot L\right)^{2}$ and ${ }^{c_{2}}=\left(K_{2} \cdot L\right)^{2}$ are constants. $\mathrm{M}$ is the number of the areas being compared.

$$
\operatorname{SSIM}_{d B}=10 \cdot \log \left(\frac{\mid \operatorname{MSSIM}_{\text {lowresolution }}-\text { MSSIM }_{\text {highresolution }} \mid}{M S S I M_{\text {lowresolution }}}\right)
$$

Because the images are of different resolution the PSNR parameter is calculated over the average power individually by the way:

$$
M S E=\left\{\frac{\left.\sum_{j=1}^{M} \sum_{k=1}^{N} I_{\text {highresolution }}(j, k)^{2}\right)}{M \cdot N}\right\}-\left\{\frac{\left(\sum_{j^{\prime}=1}^{M^{\prime}} \sum_{k^{\prime}=1}^{N^{\prime}} I_{\text {low resolution }}\left(j^{\prime}, k^{\prime}\right)^{2}\right.}{M^{\prime} \cdot N^{\prime}}\right\}
$$


$P S N R=10 \cdot \log _{10}\left(\frac{R^{2}}{M S E}\right)$

The parameter $\mathrm{R}$ is the maximum fluctuation of the input image of low resolution $\mathrm{M}, \mathrm{N}$ and $\mathrm{M}^{\prime}, \mathrm{N}^{\prime}$ are the dimensions of the high resolution and low resolution images in the process.

In Table 1 , can be seen as the amplification technique SWT- DWT- SVD $=1 \mathrm{db} 1$ has a good coefficient of conservation compared to the original image in the first iteration $n=1$, however this performance degrades significantly in a later iteration. The proposed method RHPI shows better performance than other modes of amplification in PSNR relationship and maintaining the structural similarity SSIM. In each case the present values are $59.4745 \mathrm{~dB}$ and $-57.7771 \mathrm{~dB}$. These parameters imply that the method RHPI proposed is a good interpolation technique to achieve high quality in amplification processes.

Table 1. Results Obtained in Comparing SWT- SVD- IDWT and RHPI with other Interpolation Methods

\begin{tabular}{|c|c|c|c|}
\hline $\begin{array}{c}\text { interpolation } \\
\text { methods }\end{array}$ & PSNR dB & SSIM dB & Output Dimension \\
\hline $\begin{array}{c}\text { SWT-DWT-SVD } \\
\text { it=1 db1 }\end{array}$ & 55.6692 & -61.0960 & $768 \times 1024$ \\
\hline $\begin{array}{c}\text { SWT-DWT-SVD } \\
\text { it=2 db1 }\end{array}$ & 49.1656 & -39.8000 & $1536 \times 2048$ \\
\hline Bilinear & 46.1384 & -46.3490 & $768 \times 1024$ \\
\hline Bicubic & 50.1640 & -55.0720 & $768 \times 1024$ \\
\hline Sinc & 50.1640 & -55.2314 & $768 \times 1024$ \\
\hline RHPI & 59.4745 & -57.7771 & $811 \times 1080$ \\
\hline
\end{tabular}

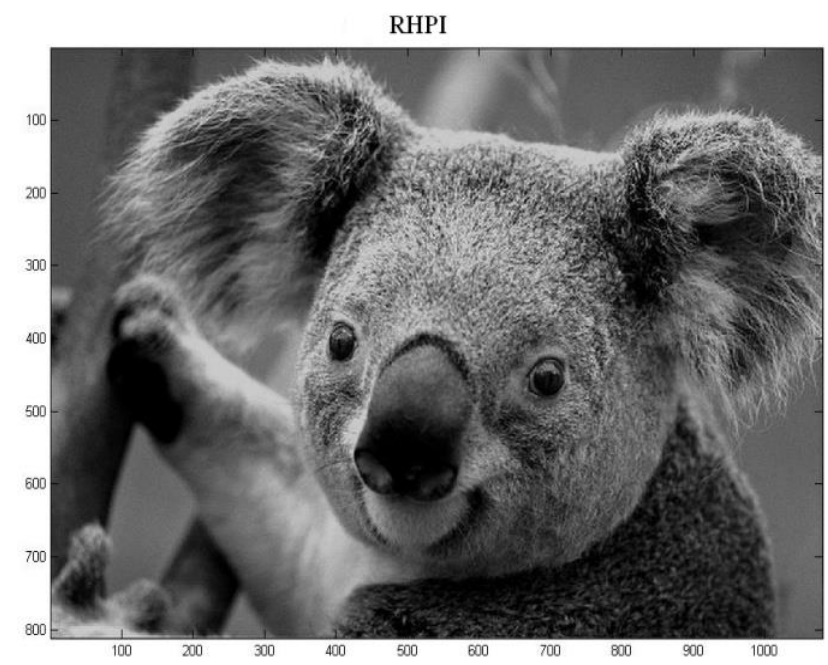



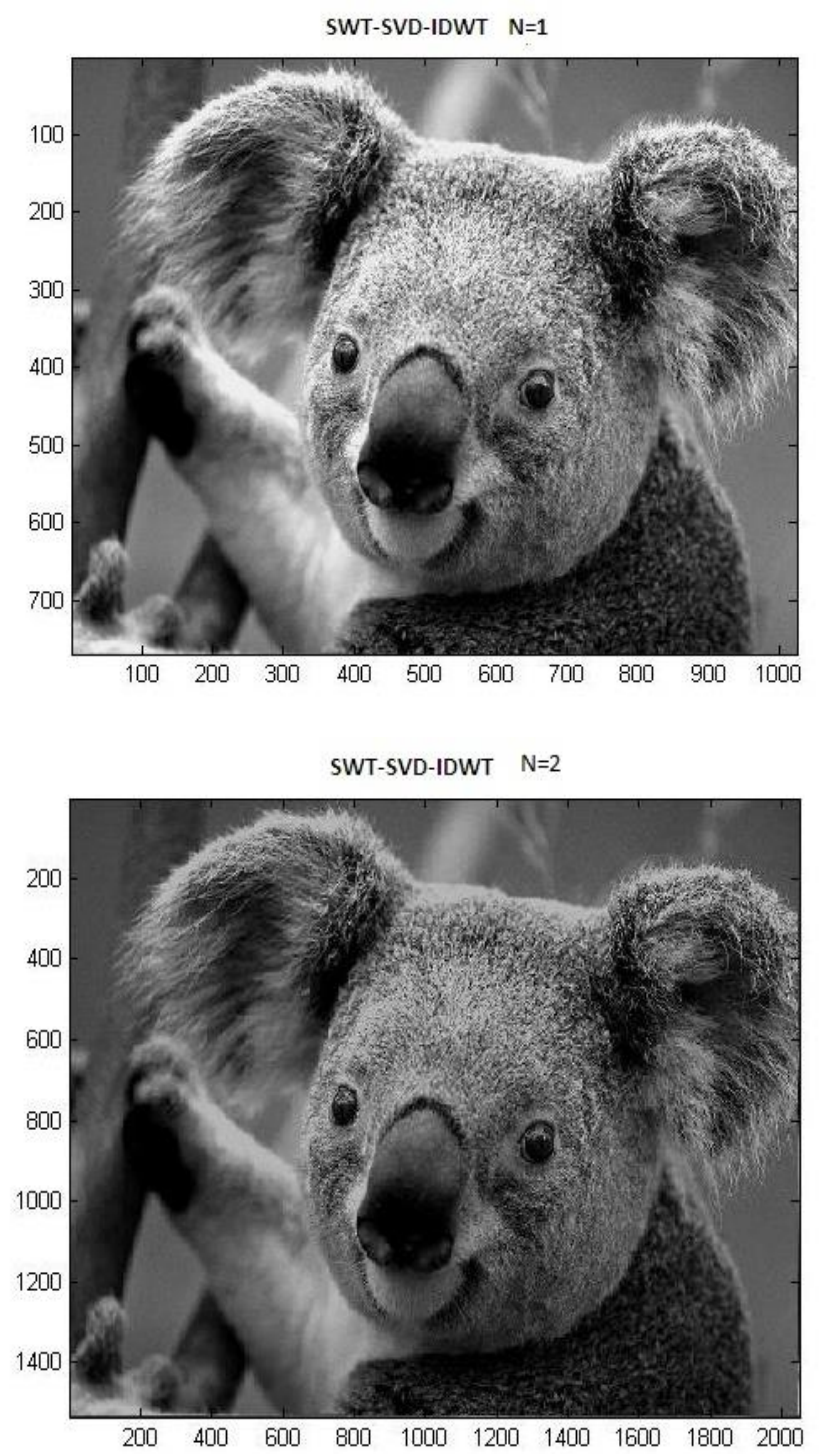

Figure 3. Images Amplified using the Proposed Procedures. From Upper to Down are Applied RHPI, SWT - IDWT $n=1$, SWT - SVD - IDWT $n=2$

\section{Conclusions}

This paper presents a novel technique for interpolation and a comparative study of the SSIM PSNR relationships with other interpolation methods. Iterative amplification by wavelet SWTSVD- IDWT presents good results using discrete transform db1. Aplying other wavelet packet the energy can be more concentrate in the image to the sub band trend. However, the method of singular value equalization is essential for the conservation of contrasts. During the first iteration of the method of wavelet exist a good relationship of SSIM but that factor for subsequent iterations decays exponentially. Moreover, the method RHPI has PSNR and SSIM indicators better than the conventional interpolation techniques or iterative amplification technique SWT-SVD-IDWT. This procedure is based on a physical model of image registration with restrictions over the kernel of interpolation. The construction of the interpolation kernel according to the high frequency content is evaluated by a Canny filter [4].The measurements obtained make the RHPI [9] method attractive for achieve high resolutions and maximum conservation of high frequencies in the output image at the plane of high resolution. 


\title{
References
}

[1] J. F. Abramatic and O. D. Faugeras, "Correction to Sequential Convolution Techniques for Image Filtering," IEEE Trans. Acoustics, Speech, and Signal Processing, ASSP, vol. 30, no. 2, (1982) April, pp. 346.

[2] G. Ambarjafari and D. Hassan, "Image super resolution based on interpolation wavelet domain high frequency sub bands and the spatial domain input image", J ETRI, vol. 32, (2010) June, pp. 390-394.

[3] J. F. Abramatic and O. Faugeras, "Design of Two-Dimensional Fir Filters from `Small Generating Kernels", IEEE Transaction on Circuits and Systems, vol. CH1318, (1978) May, pp. 116-118.

[4] J. Canny, "A Computational Approach to Edge Detection", IEEE Trans. Pattern Analysis and Machine Intelligence, PAMI-8, vol. 6, (1986) November, pp. 679-698.

[5] K. Gunaseelan and E. Seethalachmi, "Image Resolution and Contrast Enhancement Using Singular value and Discrete wavelet Decomposition”, Journal of Scientific \& Industrial Research, vol. 72, (2013) January, pp. 3135.

[6] D. Hassan and A. Gholamreza, "Image resolution enhancement by using discrete and stationary wavelet decomposition”, IEEE trans on Image process, vol. 20, (2011) October, pp. 1458-1460.

[7] D. Hassan and A. Gholamreza, "Satelite image resolution enhancement using complex wavelet transform", IEEE Geosci Remote Sensing Letter, vol. 7, (2009) September, pp. 123-126.

[8] E. Hernandez and G. Weiss, "A First Course on Wavelets, Studies in advanced mathematics", CRC Press, Boca Raton, (1996).

[9] L. Morera, "Determining parameters for images amplification by pulses interpolation", Engineering, Investigation and Technology, vol. 16, no. 1, (2015) January, pp. 73-84.

[10] A. Papoulis, "Systems and Transforms with Applications in Optics", McGraw-Hill, New York, (1966), pp. 105.

[11] K. Pratt William, "Digital Image Processing”, PIKS Inside, Third Edition. Copyright (C (2001) John Wiley \& Sons, (2001).

[12] W. K. Pratt, J. F. Abramatic and O. D. Faugeras, "Method and Apparatus for Improved Digital Image Processing", U.S. patent 4,330,833, (1982) May.

[13] C. Ramya and S. Rubba Rani, "Video denoising without motion estimation using K-mean clustering", J Sci Ind Res, vol. 70, no. 4, (2011) April, pp. 251-255.

\begin{abstract}
Author
Leandro Morera Delfin, was born in 1984; graduated of Telecommunications and Electronics Engineer and Master in Biomedical Sciences. He is actually a Professor of Communications Theory and Digital Images and Signals Processing at the Higher Polytechnic Institute Jose Antonio Echeverria, Havana, Cuba. He has job experiences in network design and software's developing for surveillance and investigations about compressing and pattern recognitions over images and videos.
\end{abstract}

